\title{
THz Generation by Optical Rectification and Competition with Other Nonlinear Processes *
}

\author{
ZHAO Zhen-Yu, HAMEAU Sophie, TIGNON Jérôme** \\ Laboratoire Pierre Aigrain, Ecole Normale Supérieure, 24 rue Lhomond, F-75005 Paris, France \\ *supported by the ENS-ECNU PhD Programme under Grant No ANR-JC05-49530, \\ ant the Laboratoire Pierre Aigrain (LPA) is the CNRS-ENS No UMR 8551 associated \\ with the Universities Paris 6 and Paris 7. \\ **Email: jerome.tignon@lpa.ens.fr \\ \%Tel: +33-1-44-32-33-54 Fax: +33-1-44-32-38-40
}

(Received 4 February 2008)

We present a study of the competition between tera-hertz $(\mathrm{THz})$ generation by optical rectification in $<110>$ ZnTe crystals, two-photon absorption, second harmonic generation and free-carrier absorption. The two-photon nonlinear absorption coefficient, second harmonic generation efficiency and free-carrier absorption coefficient in the THz range are measured independently. The incident pump field is shown to be depleted by two-photon absorption and the THz radiation is shown to be reduced, upon focusing, by free-carrier absorption. The reduction of the generated $\mathrm{THz}$ radiation upon tight focusing is explained, provided that one also takes into account diffraction effects from the sub-wavelength $\mathrm{THz}$ source.

PACS: $75.75 .+a, 75.50 . C c$ 
Ultra-fast $\mathrm{THz}$ spectroscopy is a powerful technique for studying a wide variety of materials including gases, liquids and solids.[1] In this context, a now widely used technique consists in generating $\mathrm{THz}$ pulses by optical rectification of femtosecond laser pulses.[2-4] An ultra-fast optical pulse is focused on a nonlinear crystal, which then radiates a $\mathrm{THz}$ pulse with duration of few cycles of the electromagnetic field. In the spectral domain, the radiation is broadband, from about $100 \mathrm{GHz}$ to typically 3 $\mathrm{THz}$ for a $100 \mathrm{fs}$ pulse. For an excitation with a typical Ti:sapphire mode-locked laser, the generated $\mathrm{THz}$ power is in the range of only a few tens of $\mathrm{nW}$, which stresses the importance of understanding and optimizing the THz generation in nonlinear crystals. Efficient optical rectification requires using materials with large second-order nonlinear susceptibilities and well-suited phase-matching properties for $\mathrm{THz}$ generation. ZnTe crystals with a $<110>$ orientation offer one of the best compromises.[5,6] Unfortunately, when a ZnTe crystal is irradiated with a high-power laser pulse, other competing nonlinear processes such as second harmonic generation (SHG), two-photon absorption (TPA) and subsequent free-carriers absorption (FCA) may also occur, resulting in a decrease of the $\mathrm{THz}$ generation.[6-11] Additional competing mechanisms have also been put forward, such as the fact that upon focusing, the size of the $\mathrm{THz}$ source (the laser spot size) becomes smaller than the typical $\mathrm{THz}$ wavelength, resulting in a decrease of the $\mathrm{THz}$ generation due to diffraction.[10,12] Nevertheless, to date, existing analysis of this competition are not comprehensive and the explanations are sometimes even contradictory.[10,11] Moreover, the effect of free carrier absorption[13] of the $\mathrm{THz}$ radiation (in this 
context) was neglected in previous studies.

In the present work, we investigate the $\mathrm{THz}$ generation by optical rectification in a $<110>10 \times 10 \times 2 \mathrm{~mm}$ ZnTe crystal as a function of power, focusing and crystal orientation. The conditions for the most efficient $\mathrm{THz}$ generation are described. Upon focusing, for excitation sizes smaller that the THz wavelength, SHG, TPA as well as FCA is observed while the $\mathrm{THz}$ emission is strongly reduced. While TPA is responsible for the optical pump depletion, we find that FCA of the $\mathrm{THz}$ radiation cannot be neglected, as often assumed in the past. Both contributions are found to be of the same order of magnitude.

The excitation of the $<110>2$-mm-thick crystal was provided by a $800 \mathrm{~nm}$ Ti:sapphire laser (100 fs pulses) at $300 \mathrm{~mW}$, using a 4-cm focusing lens. The ZnTe crystal is virtually transparent in the frequency domain of interest.[9] The detection of the $\mathrm{THz}$ radiation was performed by a liquid helium cooled bolometer.

First, in order to characterize their efficiency, we measured the TPA and SHG as a function of the crystal azimuthal angle and distance from focus point. Figure 1 shows the $\mathrm{THz}$ emission (spectrally integrated) as a function of the ZnTe azimuthal angle (closed symbols). The $\mathrm{THz}$ intensity is proportional to the square of the nonlinear polarization, in which angle dependence (angle between the polarization of the optical pump beam and the [001] axis) is determined by the nonlinear susceptibility tensor.[14] Figure 1 also shows the angle dependence of the measured SHG (open symbols) and of the transmission at $800 \mathrm{~nm}$ when the crystal is placed at the laser beam waist. In principle, optical rectification, SHG as well as TPA can be responsible 
for the optical pump depletion. Nevertheless, the optical rectification efficiency is only on the order of $10^{-7}$, as already pointed out in previous studies,[11] SHG generation is also inefficient in depleting the pump, and the variations observed in transmission can only be attributed to TPA. We note that easy crystal axis determination is highly desirable when developing ultra-fast $\mathrm{THz}$ spectroscopy experiments based on optical rectification and free-space electro-optical sampling. In this case, several parameters need to be properly adjusted simultaneously (crystals orientation, pump focusing, time delay, spot overlap). In this context, Fig. 1 also shows that measuring the angle-dependence of the transmission is the simplest method for determining the best crystal orientation for optimum $\mathrm{THz}$ generation.

Competition between the nonlinearities was investigated using a open-aperture z-scan method.[11,15,16] The $\mathrm{THz}$ emission, $\mathrm{SHG}$ and transmission at $800 \mathrm{~nm}$ measured as a function of the distance $z$ between the beam waist and the crystal, along the optical axis (with an azimuthal angle corresponding to a maximum of $\mathrm{THz}$ emission). As shown in Fig. 2(a), upon focusing, the THz emission first increases. For $z<10 \mathrm{~mm}$, the $\mathrm{THz}$ emission tends to saturate. For even smaller distances, the $\mathrm{THz}$ emission drops significantly, forming a $z$-hole, as already reported. As a consequence, it is now well accepted that the optimum THz generation is obtained when the crystal is placed slightly out of focus. We note that, as shown by Dakovski et al.,[10] this z-hole does not depend on the $\mathrm{THz}$ wavelength (so the fact that the emission is spectrally integrated does not play a significant role here). Figure 2(b) shows the measured SHG power and transmission at $800 \mathrm{~nm}$ as a function of distance from 
focus. TPA is responsible for the depletion of the optical pump intensity following $d I / d z=-\beta I^{2}$, where $\beta$ is the nonlinear absorption coefficient (the linear absorption is neglected). The integration of this equation leads to the $z$-dependence of the transmitted power $P_{t}=I \times S(z)=P_{0} /\left(1+\beta P_{0} S(z)^{-1} l\right)$, where $l$ is the crystal thickness and $S(z)$ the spot surface (gaussian beam). Figure 2(b) shows a fit of the transmission data giving a nonlinear absorption coefficient $\beta=4.6 \mathrm{~cm} / \mathrm{GW}$ due to TPA, in good agreement with the previous studies.[10,11,15,16]. Likewise, the SHG power is inversely proportional to the surface (Fig. 2(b)).

In order to estimate the free-carrier absorption of the $\mathrm{THz}$ radiation, we performed a two-colour experiment as depicted in Fig. 3: The ZnTe crystal is placed at $z=15 \mathrm{~mm}$ away from the focus, where optical rectification is not in competition with other nonlinearities. A BBO crystal is placed between the focusing lens and the ZnTe crystal. The $\mathrm{THz}$ radiation intensity generated by the $800 \mathrm{~nm}$ pump beam is measured when the blue-beam $(400 \mathrm{~nm})$ power is varied from $0 \mathrm{~mW}\left(I_{T H z}^{1}\right)$ to $1 \mathrm{~mW}$ $\left(I_{T H z}^{2}\right)$ by adjusting the BBO azimuthal angle. This allows extracting a free-carrier absorption coefficient $\alpha_{T H z}=l^{-1} \ln \left(I_{T H z}^{1} / I_{T H z}^{2}\right)=6 \mathrm{~cm}^{-1}$. This two-colour experiment allows estimating the effect of the free-carrier absorption on the $\mathrm{THz}$ radiation within experimental conditions as close as possible to what occurs when focusing the pump beam (i.e. when free carriers can be generated). In this case, hot free-carriers (electron and holes) are generated simultaneously with generation of the $\mathrm{THz}$ radiation (i.e. zero time-delay), resulting in absorption of the THz radiation. $[8,13]$

In the following, we first discuss the $\mathrm{THz}$ emission $z$-dependence induced by 
diffraction effects. When $z$ is large compared to the pump beam Rayleigh length, the spot surface $S$ is proportional to $z^{2}$. As pointed out in Refs. [10,12], since optical rectification is a second-order nonlinear process, the nonlinear polarization is proportional to the optical intensity $I_{0}=P_{0} / S$. When diffraction effects can be neglected (spot size larger than the typical THz wavelength, $300 \mu \mathrm{m}$ ), the THz power is $P_{T H z} \propto S\left(P_{0} / S\right)^{2} \propto z^{-2}$, as observed experimentally and depicted in Fig. 4. On the contrary, when the pump size is smaller than the $\mathrm{THz}$ wavelength, the $\mathrm{THz}$ emission from the whole emitter section interfere constructively and the $\mathrm{THz}$ emitted power reads[6] $P_{T H z} \propto\left(S\left(P_{0} / S\right)\right)^{2} \propto z^{0}$ and the THz power becomes independent of the spot size close to focus. This is sketched by the solid horizontal line in Fig. 4. Next, as shown above the $\mathrm{THz}$ radiation is partly reduced by FCA. Free carriers can be generated either by SHG or TPA in the ZnTe crystal. The free carrier generation rate by TPA is given by $\beta I_{800 \mathrm{~nm}}^{2} / 2 \hbar \omega$, whereas the free carrier generation rate by SHG is $\alpha I_{400 \mathrm{~nm}} / \hbar \omega$, where $\alpha$ is the absorption coefficient at $400 \mathrm{~nm}\left(33 \mathrm{~cm}^{-1}\right) .[11]$ As a result, the TPA over SHG free carrier generation rate ratio is 0.35 . In this context, the equation describing the $\mathrm{THz}$ generation has the form $d E_{T H z} / d z=a E_{0}^{2}-\alpha_{T H z} E_{T H z}$, where the first term is due to optical rectification and the second to the free-carrier absorption. The $\mathrm{THz}$ electric field then varies as $E_{T H z}=\eta \times a E_{0}^{2} l$, where $\eta=\left(1-e^{-\alpha_{T H z} l}\right) / \alpha_{T H z} l$ is a factor that reduces the $\mathrm{THz}$ field induced by optical rectification. If we first assume that the absorption coefficient $\alpha_{T H z}$ is proportional to the free-carrier density and thus inversely proportional to the pump spot surface, one can estimate the $z$-dependence of the $\mathrm{THz}$ radiation power in the presence of FCA 
(dotted line in Fig. 4). Nevertheless, when focusing, the pump surface becomes considerably smaller than the THz spot size. Thus, the dotted line in Fig. 4 tends to overestimate the effect of FCA. As an upper bound, one can alternatively consider that the absorption of $\mathrm{THz}$ becomes surface-independent in the diffraction limit, which underestimates FCA (dashed line). Lastly, as shown above, TPA is responsible for the depletion of the pump beam and, in the diffraction limit, this leads to a $\mathrm{THz}$ power $P_{T H z} \propto P_{0}^{2} /\left(1+\beta P_{0} S(z)^{-1} l\right)^{2}$, which is partly responsible for the formation of a z-hole close to focus. This is shown by the thick solid line in Fig. 4, where, for sake of clarity, the effect of the pump depletion by TPA on the THz emission was estimated independently of FCA.

In conclusion, we have studied the competition between $\mathrm{THz}$ generation by optical rectification, optical pump depletion by TPA and THz free-carrier absorption. We have shown that both TPA and free-carrier absorption strongly contribute to the reduction of the $\mathrm{THz}$ generation upon tight focusing. 


\section{References}

[1] D Mittleman 2003 Sensing with Terahertz Radiation ( Berlin: Springer)

[2] Auston D H 1984 Phys. Rev. Lett. 531555

[3] Xu L, Zhang X C, and Auston D H 1992 Appl. Phys. Lett. 611784

[4] D Grischkowsky 1993 Frontiers in Nonlinear Optics (Philadelphia: Institute of Physics) p 196

[5] Wu Q and Zhang X C 1996 Appl. Phys. Lett. 681604

[6] Nahata A, Weiling A S and Heintz T F 1996 Appl. Phys. Lett. 692321

[7] Zhang X C, Hu B B, Darrow J T and Auston D H 1990 Appl. Phys. Lett. 561011

[8] Sun F G and Zhang X C 2000 Conference on Lasers and Electro-Optics (CLEO), OSA Technical Digest (Washington DC: OSA) p 479

[9] Schall M and Jepsen U 2002 Appl. Phys. Lett. 804771

[10] Dakovski G, Kubera B and Shan J 2005 J. Opt. Soc. Am. B 221667

[11] Gaivoronskii V Y, Nazarov M M, Spapozhnikov D A, Shepelyavyi E V, Shkelnyuk S A, Shkurinov A P and Shuvaev A V 2005 Quant. Electron. 35407

[12] Xu J Z and Zhang X C 2002 Opt. Lett. 271067

[13] Yu P Y and Cardona M 1999 Fundamentals of Semiconductors (Berlin: Springer)

[14] Chen Q, Tani M, Jang Z P and Zhang X C 2001 J. Opt. Soc. Am. B 18823

[15] Said A A, Sheik-Bahae M, Hagan D J, Wei T H, Wang J, Young J and Van Stryland E W 1992 J. Opt. Soc. Am. B 9405

[16] He W Q, Gu C M and Shen W Z 2006 Opt. Express. 145476 


\section{Figures Caption}

Fig. 1. Closed squares: THz intensity as a function of the ZnTe azimuthal angle. Open circles: SHG intensity upon focusing. Open squares: transmission at $800 \mathrm{~nm}$.

Fig. 2. (a) THz intensity as a function of the distance to focus point (open aperture z-scan). Pump power $300 \mathrm{~mW}$. (b) Circles: SHG power. Squares: transmission at 800 nm. Solid line, right axis: fit of the TPA equation (nonlinear absorption coefficient $=4.6 \mathrm{~cm} / \mathrm{GW})$. Solid line, left axis: fit of the SHG.

Fig. 3. Scheme of the two-colour experiment. The ZnTe crystal is placed at $\mathrm{z}=15$ mm away from the focus. A BBO crystal is placed between the focusing lens and the ZnTe crystal. The $\mathrm{THz}$ radiation intensity generated by the $800 \mathrm{~nm}$ pump beam is measured as a function of the blue-beam power generated by the BBO crystal.

Fig. 4. Circles: measured $\mathrm{THz}$ intensity. Dot-dot-dashed line: expected $\mathrm{THz}$ emission z-dependence for large $z$, when the $\mathrm{THz}$ source is larger than the typical $\mathrm{THz}$ wavelength. Horizontal solid line: expected $\mathrm{THz}$ emission $z$-dependence in the diffraction limit (small excitation spot size). Solid curve: THz emission z-dependence including TPA only. Dashed line: THz emission z-dependence including FCA only, when the absorption coefficient is assumed to be independent of size. Dotted line: $\mathrm{THz}$ emission z-dependence including FCA only, when the absorption coefficient is assumed to be inversely proportional to the pump spot size. 


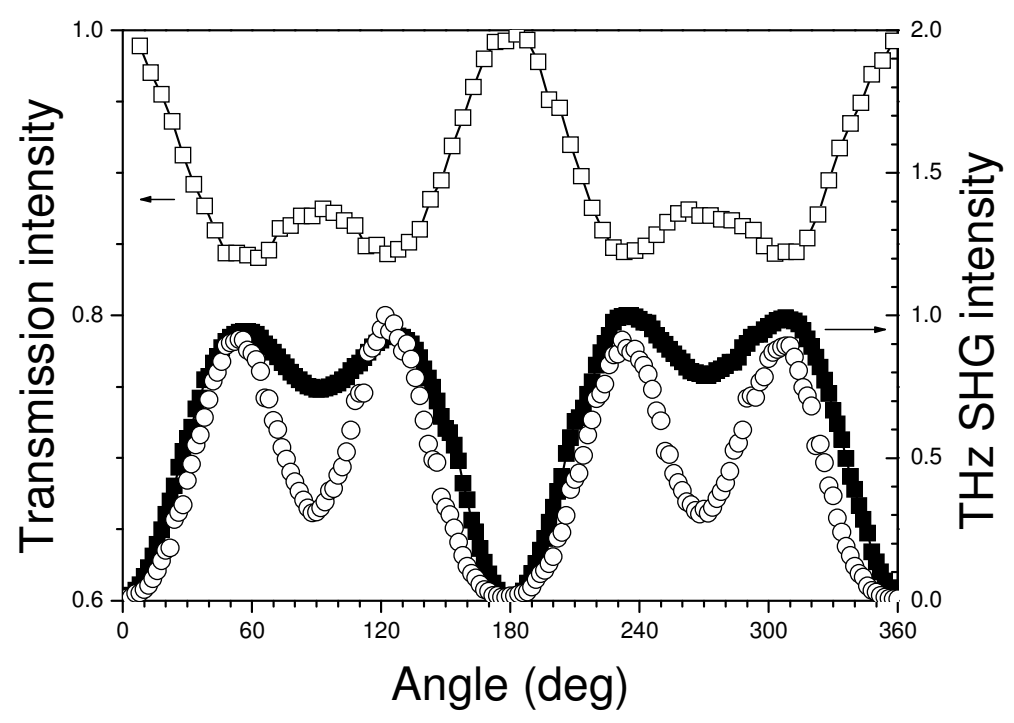

Fig. 1.

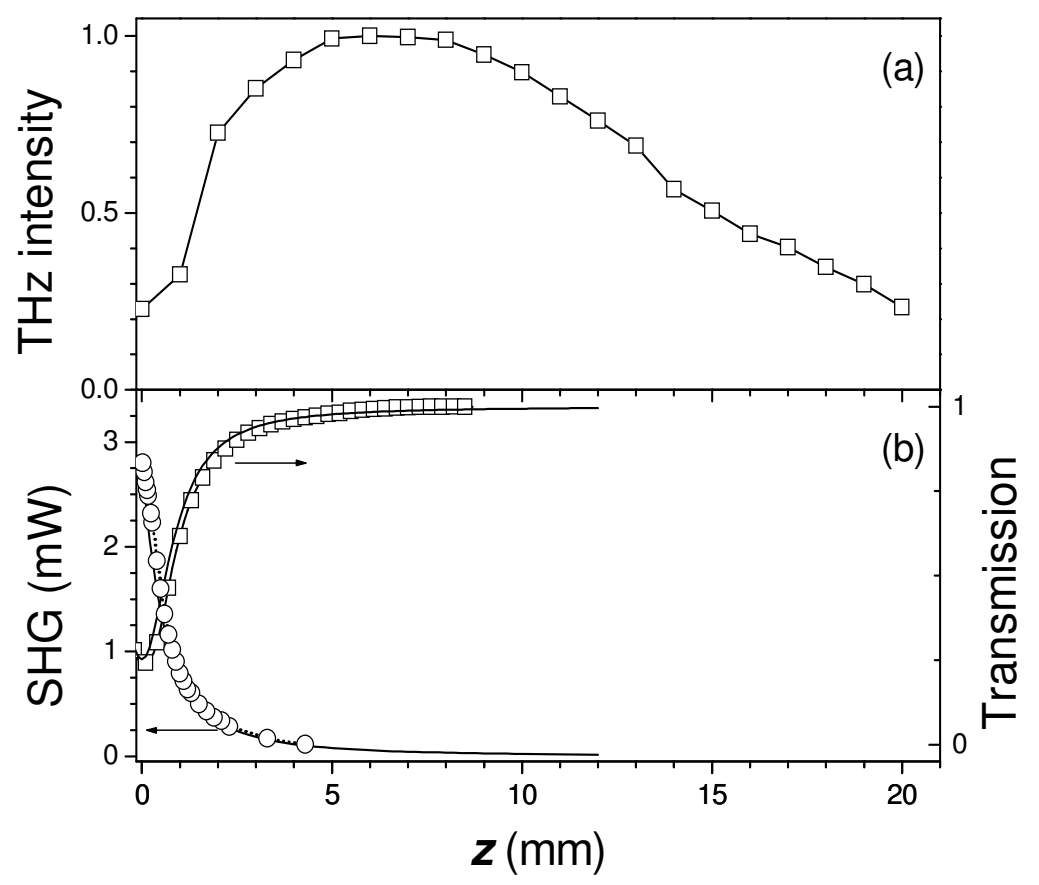

Fig. 2 . 


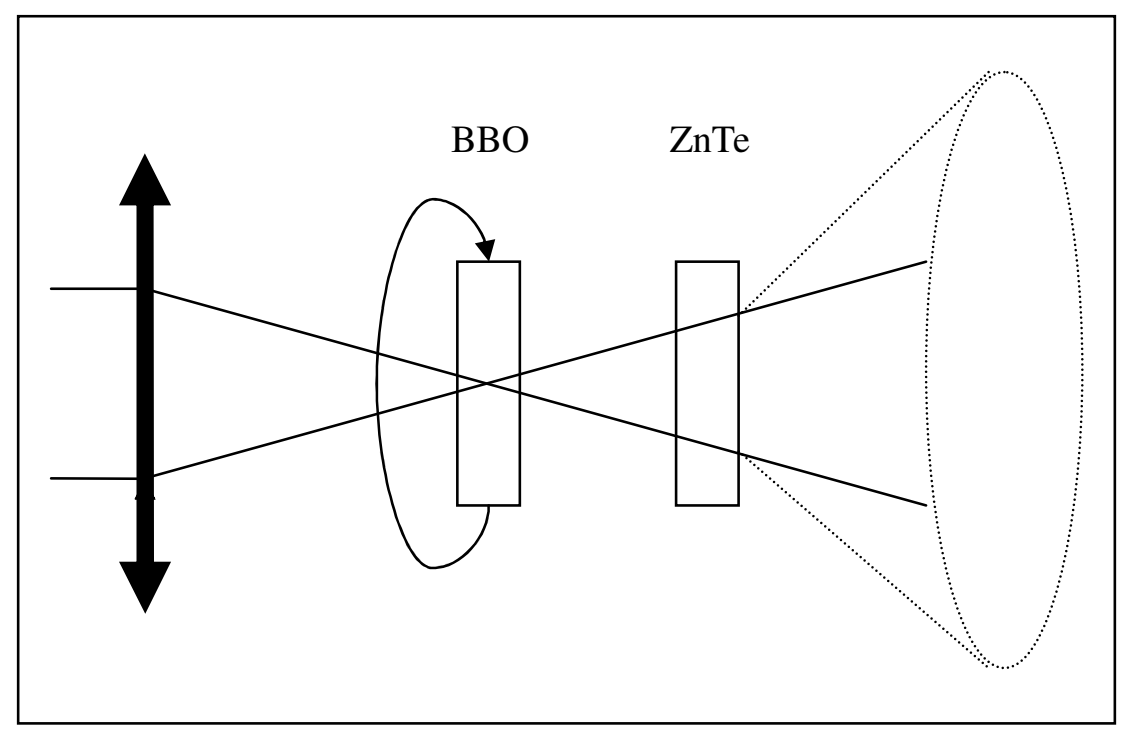

Fig. 3.

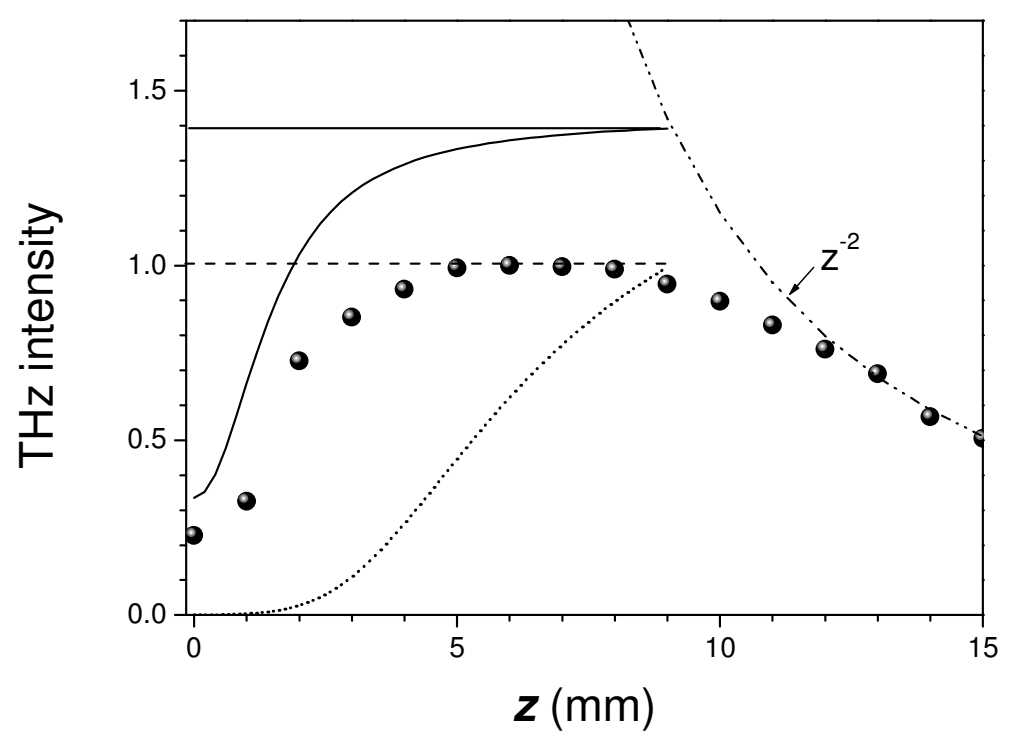

Fig. 4. 